\title{
An alternative aerosol delivery system for amiloride
}

\author{
Mark L Everard, Sunalene G Devadason, V B Sunderland, Peter N Le Souef
}

\begin{abstract}
Background - The advent of novel treatments such as aerosolized amiloride are potentially useful additions to the therapeutic options available for the treatment of cystic fibrosis. Unfortunately, amiloride and other aerosolized drugs such as antibiotics are generally administered via jet nebulisers which are time consuming to use, and thus limit the acceptance and efficacy of these forms of treatment. In vitro experiments were performed in order to determine whether amiloride could be administered in dry powder form using a Turbohaler.
\end{abstract}

Methods - Amiloride was micronised and loaded into $200 \mu \mathrm{g}$ Turbohalers. The dose distribution of the generated aerosol were assessed using a flow of $60 \mathrm{l} / \mathrm{min}$ through the Turbohaler. The dose of amiloride delivered was measured by collecting the aerosol on a filter and the quantity of drug was assayed by an ultraviolet spectrophotometric method. The particle size distribution was assessed using a Malvern MasterSizer laser particle sizer and compared with that generated by a commercially available $200 \mu \mathrm{g}$ budesonide Turbohaler.

Results - The mean (SD) dose delivered per actuation was $246 \cdot 3(40 \cdot 4) \mu \mathrm{g}$. The volume median diameter of the amiloride aerosol was $3.80(0.68) \mu \mathrm{m}$ compared with 3.07 (1.47) $\mu \mathrm{m}$ for budesonide.

Conclusions - These results suggest that therapeutic doses of micronised amiloride could be delivered effectively and conveniently as a dry powder aerosol using a Turbohaler.

(Thorax 1995;50:517-519)

Keywords: dry powder inhaler, Turbohaler, amiloride, cystic fibrosis.

for Children

M L Everard

$P$ N Le Souef

School of Pharmacy, Curtin University

V B Sunderland

Perth, Western

Australia

Reprint requests to Dr M L Everard,

Department of

Paediatrics, Sheffield

Children's Hospital,

Sheffield S10 2TH, UK.

Received 1 August 1994

Returned to authors

26 October 1994

Revised version received

9 January 1995

9 January 1995

12 January 1995

The principal attraction of delivering drugs as aerosols for the treatment of pulmonary disease is that the drugs are delivered directly to the site of action, thus reducing the possibility of systemic side effects and permitting the use of drugs that are poorly absorbed by the gastrointestinal tract or extensively metabolised by the liver. There are, however, a number of disadvantages with this route of administration, including the high variability in dose delivered to the lungs and the inconvenience of relatively long treatment times associated with the use of jet nebulisers. For patients with cystic fibrosis, delivered per actuation and particle size

via this route, the time consuming nature of nebuliser therapy is a major problem ${ }^{1}$ and may be an impediment to good compliance. The list of drugs delivered as aerosols that may be of benefit in patients with cystic fibrosis is continually growing. In addition to bronchodilators and nebulised antibiotics, novel therapies such as amiloride ${ }^{2-4}$ and DNase ${ }^{56}$ are also currently delivered via jet nebulisers. Multicentre clinical trials are under way using nebulised amiloride ${ }^{4}$ and further studies are investigating the possible benefits of administering amiloride with inhaled uridine 5triphosphate (UTP). ${ }^{7}$ However, because of its short duration of action ${ }^{8}$ it is currently administered at least four times per day, ${ }^{34}$ and hence any potential benefits are likely to be lost in many patients due to poor compliance.

It has previously been shown that micronised gentamicin could be administered as a dry powder aerosol using a Rotahaler, ${ }^{9}$ a much more convenient form of administration than a jet nebuliser. The availability of such a formulation for inhaled antibiotics would be welcomed by patients since treatment times would be reduced. This principle of using powder inhalers could be applied not only to currently used medication, but also to novel therapies such as amiloride. Dry powder inhalers are not only more convenient but there is also evidence that certain powder inhalers such as the Turbohaler (Astra Draco, Lund, Sweden) are more efficient than most jet nebulisers, providing adequate inspiratory flows can be generated.

A series of in vitro experiments was carried out in order to determine whether therapeutic doses of amiloride might be delivered quickly and conveniently using a Turbohaler.

\section{Methods}

Amiloride hydrochloride powder $(5 \mathrm{~g})$ was micronised using a Chrispro microniser (Chrispro Ltd, Canterbury, Kent, UK) at a mill pressure of $689.4 \mathrm{kPa}$ and feed pressure of $206.8 \mathrm{kPa}$. who may need to administer several drugs daily $100 \mathrm{mg}$ of the micronised amiloride. periments were carried out to assess particle size distribution and dose delivered using a "spaceship" (Astra Draco, Sweden). This simple device encloses the Turbohaler in such a way as to permit air to flow through the dosing channels and entrainment ports and out through the mouthpiece. A large three way tap permitted compressed air to be directed through the "spaceship" and hence the Turbohaler as required (fig 1 ). A flow of $60 \mathrm{l} / \mathrm{min}$, calibrated using a rotameter (Fischer and Porter Ltd, UK), was used for all experiments. 


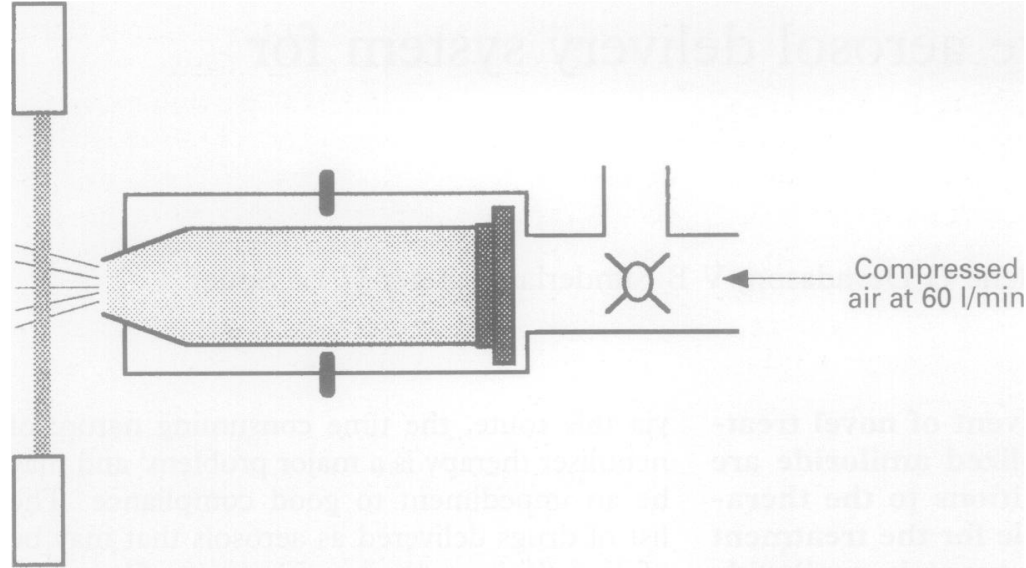

Figure 1 Particle sizing using laser particle sizer. The Turbohaler is supported in a "spaceship" permitting air to flow freely into the "spaceship", through the dosing channels and entrainment ports of the Turbohaler, and out via the spiral mouthpiece. The pattern of airflow is unaltered by the "spaceship". Compressed air flowing at $60 \mathrm{l} / \mathrm{min}$ can be directed through the Turbohaler, when required, using a large three way tap.

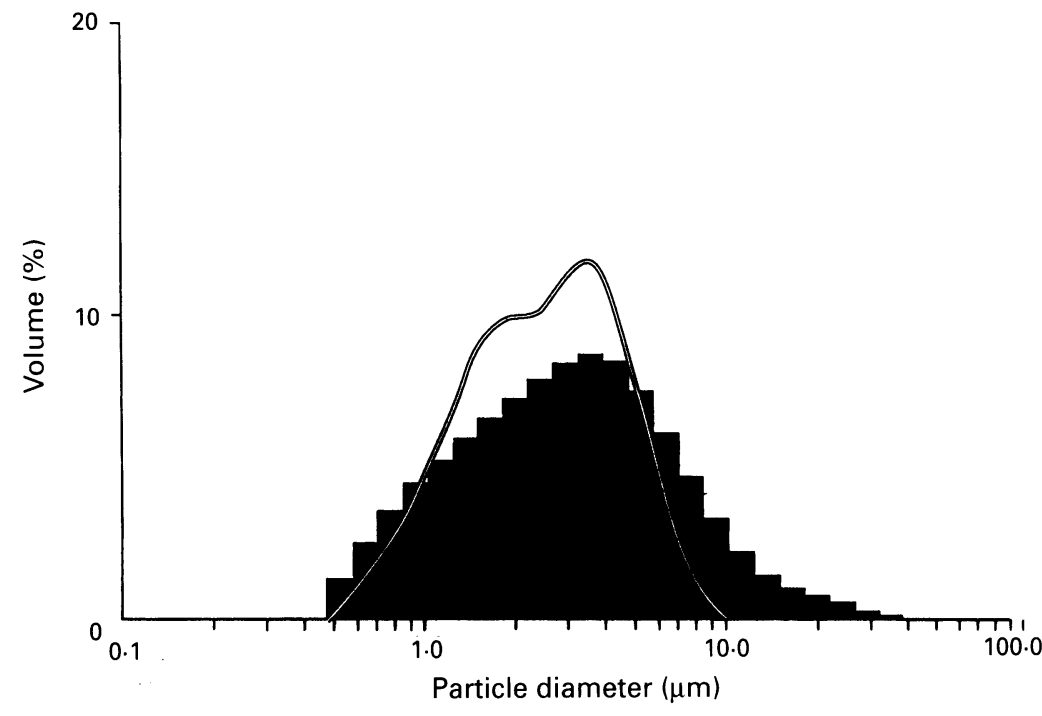

Figure 2 Particle size distribution of aerosol generated by an amiloride-containing Turbohaler (histogram) compared with that from a budesonide-containing Turbohaler (continuous line) as assessed with a Malvern MasterSizer laser particle sizer.

The spaceship also permits rotation of the Turbohaler turning grip and hence the dosing channels within the Turbohaler may be loaded without removing the Turbohaler from the spaceship.

\section{ASSESSMENT OF DOSE DELIVERED}

For each experiment the dosing channels were loaded and the powder within the dosing channels was then blown out of the Turbohaler onto a filter (Kendall Curity Anaesthesia Filter). Ten doses were delivered for each experiment. Seven experiments were performed using the amiloride-filled Turbohalers and $10 \mathrm{ex}-$ periments with the commercial budesonide Turbohalers.

The amount of amiloride deposited on the filters and mouthpieces was measured using the following method. The filters and mouthpieces were washed with $100 \mathrm{ml} 0 \cdot 13 \%$ saline; $4.9 \mathrm{ml}$ of $0 \cdot 1 \mathrm{M} \mathrm{HCl}$ was added to $40 \mathrm{ml}$ of the filter wash and the solution was made up to $50 \mathrm{ml}$ with $0 \cdot 13 \%$ saline. The absorbance $(\lambda=$
Mean (SD) drug delivery from an amiloride-filled Turbohaler $(n=7)$ and a commercial budesonide Turbohaler $(n=10)$ at a flow rate of $60 \mathrm{l} / \mathrm{min}$

\begin{tabular}{|c|c|c|}
\hline & $\begin{array}{c}\text { Amiloride } \\
\text { ( } \mu \mathrm{g} / \text { dose })\end{array}$ & $\begin{array}{l}\text { Budesonide } \\
\text { ( } \mu \text { g/dose) }\end{array}$ \\
\hline $\begin{array}{l}\text { Mouthpiece } \\
\text { Filter } \\
\text { Total }\end{array}$ & $\begin{array}{r}17.8(6.5) \\
246.3(40.4) \\
264 \cdot 0(41 \cdot 2)\end{array}$ & $\begin{array}{r}61 \cdot 5(14 \cdot 0) \\
142.6(16 \cdot 4) \\
204 \cdot 0(19 \cdot 6)\end{array}$ \\
\hline
\end{tabular}

$362 \mathrm{~nm}$ ) of each sample was measured in duplicate on a spectrophotometer (Hitachi U- $\frac{O}{\bar{S}}$ 2000). The concentration of amiloride in the filter and mouthpiece washes was calculated using the extinction coefficient of amiloride in $s$ $0 \cdot 13 \%$ saline $(\varepsilon 1 \% 1 \mathrm{~cm}=610)$. The amount $\vec{\circ}$ of amiloride ( $\mu \mathrm{g} /$ dose) could then be calculated $\vec{\omega}$ for each of the washes. A maximum error of $\stackrel{\omega}{\mathcal{L}}$ $3.4 \%$ was obtained when a known con- $\overrightarrow{\vec{x}}$ centration of amiloride was assayed using this method.

The quantity of budesonide was measured or as follows. The filters and mouthpieces were $\vec{V}$ washed with approximately $40 \mathrm{ml}$ ethanol and the volume of each wash was then made up to $\vec{r}$ a total of $50 \mathrm{ml}$ with ethanol. The absorbance $(\lambda=243 \mathrm{~nm})$ of each sample was measured in duplicate on a spectrophotometer (Hitachi U- $\bullet$ 2000). The concentration of budesonide in each of the washes was calculated using the absorbance of a solution containing a known concentration of budesonide. The standard curve for budesonide was linear $\left(r^{2}=1.00\right)$ for $\frac{2}{D}$ concentrations between 1 and $30 \mathrm{mg} / \mathrm{ml}$.

\section{ASSESSMENT OF PARTICLE SIZE}

The sizes of particles from the amiloride-filled $\vec{\partial}$ Turbohalers were measured using a Malvern MasterSizer X laser particle size analyser (Mal- $\dot{\sigma}$ vern Instruments Ltd, UK). Turbohalers were placed in a "spaceship" and the micronised amiloride powder blown across the path of the laser. For comparison, the particle size 음 distribution from the $200 \mu \mathrm{g}$ commercial bu- $\rightarrow$ desonide Turbohaler was measured using the same method. This approach to particle sizing $N$ aerosols generated by dry powder inhalers has been used in previous studies. ${ }^{1011}$

\section{Results}

The mean total output from both the amiloride- $T$ filled Turbohaler and the commercial $(200 \mu \mathrm{g}) \stackrel{\overrightarrow{\mathrm{O}}}{\mathrm{D}}$

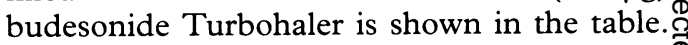
The proportion of amiloride deposited on the mouthpiece was much lower than that of budesonide, but the total dose delivered was 0 higher for amiloride. The variability in the dosec of amiloride delivered was greater than that of budesonide.

The mean (SD) volume median diameter of the dose delivered from the amiloride-filled Turbohaler was $3.80(0.68) \mu \mathrm{m}$ compared with $3.07(1.47) \mu \mathrm{m}$ from the commercial budesonide Turbohaler. The particle size distribution of the aerosols generated by the amiloride and budesonide-containing Turbohalers is illustrated in fig 2 . 


\section{Discussion}

These results suggest that doses of amiloride, similar to those delivered via jet nebulisers, could be delivered efficiently to the lungs of patients with cystic fibrosis using a Turbohaler.

The results obtained using the Malvern MasterSizer show that the particle size distribution obtained using amiloride was similar to that generated by commercial Turbohalers containing budesonide. The slightly broader spread of particle sizes and slightly larger mass median diameter are likely to be related to the microniser used. Although this method for particle sizing aerosols generated by Turbohalers has been used before, ${ }^{1011}$ the figures for volume median diameter cannot be used to calculate the mass median aerosol diameter since the particles are not spheres. Hence the results presented here illustrate qualitative changes in the aerosol characteristics and not quantitative changes in the mass median aerosol diameter.

The dose delivered from the amiloride Turbohaler was more than $200 \mu \mathrm{g}$ while the variability in the dose delivered was also greater. This is probably because we used micronised, non-spheronised, powder which will be denser, leading to an increased dose, but will tend to flow less smoothly leading to greater inconsistencies in the filling of the dosing channels within the Turbohaler. Reproducibility might be increased if the powder could be spheronised.

A recent radiolabelled deposition study using jet and ultrasonic nebulisers ${ }^{12}$ found that the mean dose of amiloride delivered to the lungs of patients with cystic fibrosis from a $1 \mathrm{mg}$ initial dose was $103 \mu \mathrm{g}$ using an ultrasonic nebuliser and $53 \mu \mathrm{g}$ with a jet nebuliser. As in previous studies with nebulisers a wide range was observed in the dose delivered to the lungs. A recent radiolabelled deposition study suggested that approximately $27 \%$ of the budesonide contained in a $200 \mu \mathrm{g}$ Turbohaler might be delivered to the lungs of healthy individuals when inhaling at approximately $601 / \mathrm{min}$ through the device, ${ }^{13}$ giving a lung dose of $54 \mu \mathrm{g}$. Our data suggest that similar results could be obtained when using Turbohalers containing amiloride. If higher lung doses were deemed desirable, it would be a simple matter to take a second dose from the Turbohaler or, alternatively, devices dispensing 400 or $500 \mu \mathrm{g}$ could be used.

As with any dry powder inhaler, the efficiency of such a delivery system would obviously be limited in very young patients and those with extensive disease since the efficiency of delivery to the lower respiratory tract is determined to a large extent by the inspiratory effort generated by the patient. Another potential problem associated with dry powder inhalers is that current devices deliver more than $50 \%$ of the dose to the upper airway, much of which is subsequently swallowed. This may be a problem with drugs such as amiloride which are absorbed from the gastrointestinal tract. However, strategies to reduce substantially the gastrointestinal dose, such as mouth rinsing, are available.

Patients report that the daily requirement for time consuming treatments, especially when aerosols are utilised, is one of the most demanding aspects of living with cystic fibrosis. ${ }^{1}$ This study, and a previous study in which gentamicin was administered via the Rotahaler, ${ }^{8}$ indicates that a wide range of drugs could be delivered to the lungs of patients with cystic fibrosis using dry powder inhalers which would substantially reduce the time taken to administer treatments. It is likely that novel aerosol delivery systems, which are both convenient and more efficient than existing devices, will become available in the near future, and some of these may be eminently suitable for the delivery of drugs used in the treatment of cystic fibrosis. Unfortunately the cost involved in obtaining approval from regulatory bodies for new formulations is considerable and the size of the potential market makes such developments unattractive for pharmaceutical companies. Development of convenient aerosol delivery systems containing drugs such as amiloride and antibiotics will probably only occur if physicians and patient interest groups can persuade pharmaceutical companies that such developments would be of great benefit to patients with cystic fibrosis, and work with them to facilitate approval of such delivery systems.

1 Smith G. A patient's view of cystic fibrosis. F Adolescent Health Care 1986;7:134-8.

2 App EM, King M, Helfesreider R, Kohler D, Matthys H. Acute and long term amiloride inhalation in cystic fibrosis lung disease. A rational approach to cystic fibrosis therapy. lung disease. A rational approach to cysti

3 Knowes MR, Church NL, Waltner MSW, Yankaskas JR Gilligan P, King M, et al. A pilot study of aerosolized amiloride for the treatment of lung disease in cystic fibrosis. $N$ Engl f Med 1990;332:1189-94.

4 Tomkiewicz RP, App EM, Zayas JG, Ramirez O, Church $\mathrm{N}$, Boucher RC, et al. Amiloride inhalation therapy in cystic fibrosis. Influence on ion content, hydration, and

5 Hubbard RC, McElvaney NG, Birrer P, Shak S, Robinson WW, Jolley C, et al. A preliminary study of aerosolised recombinant deoxyribonuclease $I$ in the treatment of cystic fibrosis. $N$ Engl $\mathcal{F}$ Med 1992;326:812-5.

6 Ranasinha C, Assoufi B, Shak S, Christiansen D, Fuchs H, Empey D, et al. Efficacy and safety of short term administration of aerosolised recombinant human DNase I in adults with stable stage cystic fibrosis. Lancet 1993; 342:199-202.

7 Bennett WD, Olivier KN, Zeman KL, Hohneker KH, Boucher RC, Knowles MR. Peripheral lung clearance in cystic fibrosis: effect of aerosolized uridine 5-triphosphate (UTP) plus amiloride. Pediatr Pulmonol 1994; Supply 10: A213.

8 Anderson WH. Pharmacokinetics of amiloride by inhalation in adults, adolescents, and children. Pediatr Pulmonol 1993; Suppl 9:150-1.

9 Goldman JM, Bayston SM, O'Connor S, Meigh RE. Inhaled micronised gentamicin powder: a new delivery system. micronised gentamicin pow

10 Plomp A, Fonteijn PB, Andersson JAR. Effects of relative humidity on particle size distribution from Turbuhaler. In: Newman SP, Moren F, Crompton GK, eds. $A$ new concept in inhalation therapy. Bussum, The Netherlands: Medicom, 1987:100-3.

11 Olsson B, Jaegfeldt, Hed K, Lundback H. Correlation between laser scattering and inertial impaction for the particle tween listribution characteristics of Bricanyl Turbuhaler. $\mathcal{F}$ Aerosol Sci 1988;19:1109-11.

12 Thomas SHL, O'Doherty MJ, Graham A, Page CJ, Blower P, Geddes DM, et al. Pulmonary deposition of nebulised amiloride in cystic fibrosis: comparison of two nebulisers. Thorax 1991;46:717-21.

13 Borgstrom L, Bondesson E, Moren F, Newman SP. Lung deposition of budesonide inhaled via Turbuhaler. A comparison with terbutaline sulphate. Eur Respir $\mathcal{F} 1994 ; 7$ 68-73. 